Pacific Journal of Mathematic 


\section{ON SUBGROUPS OF PRIME POWER INDEX}

\section{F. HARRIS}

Let $G$ be an abelian group. A set $S \subset G$ is a stellar set if $m x \in S$ implies $x, 2 x, \cdots, m x \in S$. Let $p^{\alpha}$ be a fixed prime power. It is shown that if $S \cap p^{\alpha} G=\varnothing, G$ satisfies a mild condition, and $S$ intersects all the subgroups $K$ of index $G: K=p^{\alpha}$, then the cardinality of $S$ is bounded below by $p^{\alpha}+p^{\alpha-1}$. This bound is the best possible. The problem is reduced to solving a number of congruence relations

$$
\lambda_{1} x_{1}+\lambda_{2} x_{2}+\cdots+\lambda_{n} x_{n} \equiv 0\left(p^{\alpha}\right)
$$

with lattice points $\left(x_{1}, x_{2}, \cdots, x_{n}\right)$ in a stellar set $S$ in Euclidean $n$-space. This in turn leads to an interesting result on congruence classes of subgroups and points which tells something about the solution in integers of the above congruence relation.

G. K. White [3] has shown that if $G$ is an abelian group without elements of order $p^{\beta}, 1<p^{\beta}<p^{\alpha}$, and $S$ is a stellar set as above, then

$$
\begin{array}{ll}
|S| \geqq p^{\alpha}+p & \text { if } \alpha \geqq 2 \\
|S| \geqq p+1 & \text { if } \alpha=1 .
\end{array}
$$

( $|S|$ is the cardinal number of the set $S$.

We improve this to get

THEOREM 1. Suppose $p^{\alpha}$ is fixed, $G$ is an abelian group without elements of order $p^{\beta}, 1<p^{\beta}<p^{\alpha}$, and $S$ is a stellar set satisfying $S \cap p^{\alpha} G=\varnothing \quad$ which intersects all the subgroups $K$ of index $G: K=p^{\alpha}$. Then

$$
|S| \geqq p^{\alpha}+p^{\alpha-1}
$$

J. W. S. Cassels [1] has shown that if a stellar set $S$ intersects all the subgroups of index $\leqq m$ in an abelian group without elements of finite order than $|S| \geqq m$. Our result is an improvement for $m=p^{\alpha}$.

Let g. c. d. $\left(a_{1}, \cdots, a_{k}\right)$ denote the greatest common divisor of $a_{1}, \cdots, a_{k}$. Let $V_{\alpha}$ denote the Cartesian product of $n \geqq 1$ copies of $Z_{p^{\alpha}}$, the residue class ring modulo $p^{\alpha}$. Let $\Lambda_{0}$ denote the free abelian group of rank n. An $n$-tuple (in $\Lambda_{0}$ or in $V_{\alpha}$ ) is said to be $p$-primitive if $p$ does not divide at least one coefficient of the $n$-tuple. An integer $x$ is said to be $p$-prime if g.c.d. $(p, x)=1$. Let $V_{\alpha}^{*}$ denote the set 
of those $p$-primitive elements of $V_{\alpha}$ whose first $p$-prime coefficient is 1 .

$$
\text { If } x=\left(x_{1}, \cdots, x_{n}\right) \in \Lambda_{0} \text { and } \lambda=\left[\lambda_{1}, \cdots, \lambda_{n}\right] \in V_{\alpha}^{*}
$$

the dot product is $\lambda \cdot x=\lambda_{1} x_{1}+\cdots+\lambda_{n} x_{n}$. Because of the one-toone correspondence between $\lambda \in V_{\alpha}^{*}$ and the subgroup

$$
\left\{x \mid x \in \Lambda_{0} \text { and } \lambda \cdot x \equiv 0\left(p^{\alpha}\right)\right\}
$$

of index $p^{\alpha}$ in $\Lambda_{0}$ we may identify the two. Thus we write $x \in \lambda$ to mean $\lambda \cdot x \equiv 0\left(p^{\alpha}\right)$.

By the same reasoning as in [3], Theorem 1 follows from

THEOREM 2. Suppose that for fixed $p^{\alpha}, n \geqq 2$ every congruence $\lambda \cdot x \equiv 0\left(p^{\alpha}\right), \lambda \in V_{\alpha}^{*}$, has a solution $x$ in a stellar set $S$ satisfying $S \cap p^{\alpha} \Lambda_{0}=\varnothing$. Then $|S| \geqq p^{\alpha}+p^{\alpha-1}$.

C. A. Rogers [2] has proved Theorem 2 for the case $\alpha=1$. Two $n$-tuples $\lambda$ and $\mu$ are said to be congruent modulo $p^{r}$ if each component of $\lambda$ is congruent modulo $p^{r}$ to the corresponding component of $\mu$. If $\lambda$ and $\mu$ are $p$-primitive elements of $\Lambda_{0}$ and $\lambda \not \equiv k \mu(p)$ for all $p$-prime $k$ then

$$
\left\{x \mid x \in \Lambda_{0} \text { and } \lambda \cdot x \equiv 0\left(p^{\alpha-1}\right) \text { and } \mu \cdot x \equiv 0(p)\right\}
$$

is a subgroup of index $p^{\alpha}$ in $\Lambda_{0}$, so for $\alpha \geqq 2$ there are many more subgroups of index $p^{\alpha}$ in $\Lambda_{o}$ than those we are considering in Theorem 2. In order to prove Theorem 2 we need a result on congruence classes of subgroups and points which has some interest in its own right. If $y$ is a $p$-primitive element in a stellar set $T$ in $\Lambda_{0}$. let

$$
T(y)=\{m x \in T \mid x \equiv y(p) \text { and } m=1,2,3, \cdots\} .
$$

Then $T(y)$ is also stellar and we say $T(y)$ is a p-class of points of $T$.

THeorem 3. Suppose that $\alpha \geqq \gamma \geqq 2, n \geqq 3$ and $\lambda^{0} \in V_{\alpha}^{*}$ are fixed. If for each $\lambda$ such that $\lambda=\lambda^{\circ}(p)$ and $\lambda \in V_{\alpha}^{*}$ the congruence $\lambda \cdot x \equiv 0\left(p^{r}\right)$ has a solution $x \in T$ where $T$ is a stellar subset of $\Lambda_{0}$. satisfying $T \cap p^{\alpha} \Lambda_{0}=\varnothing$ then either ( $\mathrm{i}$ ) all the congruences have a solution in a p-class $T\left(x^{0}\right)$ of points of $T$ for some $x^{0} \in T$ and

$$
|T| \geqq\left|T\left(x^{0}\right)\right| \geqq p^{\gamma-1}
$$

or (ii) $|T| \geqq p^{\gamma-1}+\max \left(|T(x)|, p^{\gamma-2}\right)$ for all $x \in T$.

2. Lemmas. Theorem 3 is proved by induction. We need two 
lemmas for the inductive step and one for the case $\gamma=2$. Assume $\alpha \geqq \gamma$. Let $\mu \in V_{\alpha}^{*}$ and define

$$
\Lambda_{r}(\mu)=\left\{\lambda \mid \lambda \equiv \mu\left(p^{\alpha-r}\right)\right\}=\left\{\mu+\lambda p^{\alpha-r} \mid 1 \leqq \lambda_{i} \leqq p^{r}\right\} \subset V_{\alpha}^{*} .
$$

Then

$$
\begin{array}{ll}
\Lambda_{r}(\mu) \cap \Lambda_{r-1}(\nu)=\varnothing & \text { if } \mu \neq \nu\left(p^{\alpha-\gamma}\right) \\
\Lambda_{r}(\mu) \supset \Lambda_{r-1}(\nu) & \text { if } \mu \equiv \nu\left(p^{\alpha-\gamma}\right) .
\end{array}
$$

Thus

$$
\Lambda_{\gamma}(\mu)=U\left\{\Lambda_{\gamma-1}\left(\mu+\mu^{\prime} p^{\alpha-r}\right) \mid 1 \leqq \mu_{i}^{\prime} \leqq p\right\} .
$$

Since each $\Lambda_{r}(\mu)$ is a set of $\lambda \in V_{\alpha}^{*}$ and each $\lambda$ can be regarded as a set of $x \in \Lambda_{0}$, the $x$ are in some sense "second level" elements of $\Lambda_{\gamma}(\mu)$. We write $x * \Lambda_{\gamma}(\mu)$ if $x \in \lambda$ for some $\lambda \in \Lambda_{\gamma}(\mu)$.

Suppose $C$ is a family of $\Lambda_{\gamma}(\mu)$. We define ordered pairs

$$
\begin{aligned}
A(C, x) & =\left\{\Lambda_{r}(\mu) \mid \Lambda_{\gamma}(\mu) \in C \text { and } x * \Lambda_{\gamma}(\mu)\right\} \\
B\left(\Lambda_{\gamma}(\mu), x\right) & =\left\{\lambda \mid \lambda \in \Lambda_{r}(\mu) \text { and } x \in \lambda\right\} \\
B\left(\Lambda_{r}(\mu), T\right) & =\bigcup_{x \in T} B\left(\Lambda_{r}(\mu), x\right) .
\end{aligned}
$$

We say $T$ covers $\Lambda_{r}(\mu)$ if and only if $B\left(\Lambda_{r}(\mu), T\right)=\Lambda_{r}(\mu)$.

We wish to cover $\Lambda_{r}\left(\lambda^{\circ}\right)$. Without loss generality take $\lambda^{\circ}=[1,0, \cdots, 0]$ and let $\Lambda_{\gamma}=\Lambda_{\gamma}\left(\lambda^{\circ}\right)$. Now $x * \Lambda_{\gamma}$ if and only if

$$
\lambda \cdot x=\left(\lambda^{\circ}+p^{\alpha-r} \lambda\right) \cdot x \equiv 0\left(p^{\alpha}\right)
$$

for some

$$
\lambda^{\circ}+p^{\alpha-\gamma} \lambda=\left[1, \lambda_{2} p^{\alpha-\gamma}, \cdots, \lambda_{n} p^{\alpha-\gamma}\right] \in \Lambda_{\gamma} .
$$

This implies

$$
\begin{aligned}
\lambda^{\circ} \cdot x & \equiv w_{1} p^{\alpha-\gamma}\left(p^{\alpha}\right) \quad \text { for some } w_{1} \\
w_{1}+\lambda \cdot x & \equiv 0\left(p^{r}\right) \\
w_{1}+\sum_{2}^{n} \lambda_{i} x_{i} & \equiv 0\left(p^{r}\right)
\end{aligned}
$$

Thus $T$ covers $\Lambda_{r}$ if and only if the congruence (1) is satisfied for all $\left[1, \lambda_{2}, \cdots, \lambda_{n}\right]$ by points $\left(p^{\alpha-\gamma} w_{1}, x_{2}, \cdots, x_{n}\right) \in T$. By $\left(^{*}\right)$ we may write (1) as

$$
w_{1}+\sum_{2}^{n}\left(\mu_{i}^{\prime}+\nu_{i} p\right) x_{i} \equiv 0\left(p^{r}\right)
$$

and $T$ covers $\Lambda_{\gamma-1}\left(\lambda^{\circ}+\mu^{\prime} p^{\alpha-\gamma}\right)$ if and only if (2) is satisfied for all $\nu_{i}$. To simplify notation let $\Lambda\left(\mu^{\prime}\right)=\Lambda_{r-1}\left(\lambda^{0}+\mu^{\prime} p^{\alpha-\gamma}\right)$.

$$
\text { Since } \lambda^{\circ}=[1,0, \cdots, 0] \text { implies } x_{1}^{0} \equiv 0(p),\left(x_{k}^{0}, p\right)=1
$$

for some $k>1$, without loss of generality take $k=n, x_{n}^{0}=1$ and 
a suitable coordinate transformation will take $x^{0}$ into $(0, \ldots, 0,1)$ but leave $\lambda^{\circ}=[1,0, \cdots, 0]$ fixed. Thus we shall work with

$$
\begin{aligned}
T_{*} & =T(0, \cdots, 0,1)=T\left(x^{0}\right) \\
\Lambda_{\gamma} & =\Lambda_{r}([1,0, \cdots, 0])
\end{aligned}
$$

but our results hold for all $T(x)$ and $\Lambda_{r}(\mu)$. Now $x \in T_{*}$ and $x * \Lambda_{r}$ implies

$$
w_{1}+p \sum_{2}^{n-1}\left(\mu_{i}^{\prime}+\nu_{i} p\right)+\mu_{n}^{\prime}+\nu_{n} p \equiv 0\left(p^{r}\right)
$$

so $x \in T_{*}$ and $x * \Lambda\left(\mu^{\prime}\right)$ if and only if

$$
w_{1}+\mu_{n}^{\prime} \equiv 0(p) \text {. }
$$

Because of (4) we can define subsets $T_{C}$ of $T_{*}$ which are in $\Lambda\left(\mu^{\prime}\right)$. At the same time we define families of congruence classes $\Lambda\left(\mu^{\prime}\right) \subset \Lambda_{\gamma}$ which we shall need for the lemmas. In the following $c=1, \cdots, p$.

$$
\begin{aligned}
T_{c} & =\left\{m x \in T_{*} \mid x=\left(c p^{\alpha-\gamma}+x_{1} p^{\alpha-\gamma+1}, x_{2} p, \cdots, x_{n-1} p, 1\right),\right. \\
M_{c} & =\left\{\Lambda\left(\mu^{\prime}\right) \subset \Lambda_{r} \mid \mu_{n}^{\prime}+c \equiv 0(p)\right\} \\
Q^{\prime} & =\left\{M_{c} \mid B\left(\Lambda\left(\mu^{\prime}\right), T_{*}\right)=\Lambda\left(\mu^{\prime}\right) \text { for some } \Lambda\left(\mu^{\prime}\right) \in M_{c}\right\} \\
R^{\prime} & =\left\{M_{c} \mid M_{c} \notin Q^{\prime}\right\} \\
Q & =\cup\left\{\Lambda\left(\mu^{\prime}\right) \in M_{c} \mid M_{c} \in Q^{\prime}\right\} \\
R & =\bigcup\left\{\Lambda\left(\mu^{\prime}\right) \in M_{c} \mid M_{c} \in R^{\prime}\right\} \\
P & =Q \cup R=\left\{\Lambda\left(\mu^{\prime}\right) \subset \Lambda_{r}\right\}
\end{aligned}
$$

$T_{c} \subset \Lambda_{0} ; M_{c}$ is a collection of classes $\Lambda\left(\mu^{\prime}\right)$, etc.

Notice that if $\Lambda\left(\mu^{\prime}\right) \in R$ then $B\left(\Lambda\left(\mu^{\prime}\right), T_{*}\right) \neq \Lambda\left(\mu^{\prime}\right)$, but the converse is not necessarily true. Also $T_{*}$ is the disjoint union

$$
T_{*}=\bigcup_{c=1}^{P} T_{c}
$$

and $P$ is the disjoint union of $Q$ and $R$. Hereafter suppose

$$
\left|T_{*}\right|<p^{r}
$$

and

$$
(0,0, \cdots, 0) \notin T_{*}
$$

LeMma 1. (a) If $\mu_{n}^{\prime} \neq-c(p)$ then $B\left(\Lambda\left(\mu^{\prime}\right), T_{c}\right)=\varnothing$.

(b) If $T_{*}$ covers a $\Lambda\left(\mu^{\prime}\right)$ then

$$
\left|T_{c}\right| \geqq p^{\gamma-1} \text { and } c+\mu_{n}^{\prime} \equiv 0(p) .
$$

(c) If the $\Lambda\left(\mu^{\prime}\right)$ covered are from $<$ distinct $M_{c},\left(0 \leqq \measuredangle=\left|Q^{\prime}\right|<p\right)$ then 


$$
\begin{aligned}
& \left|T_{*}\right| \geqq \ell p^{\gamma-1} \\
& |Q|=\ell p^{n-2}
\end{aligned}
$$

and

$$
|R|=p^{n-1}-\ell p^{n-2} .
$$

Proof. (a) follows from (4).

(b) Define a set $V_{c}$, not stellar, by

$$
V_{c}=\left\{p^{\beta} y \in T_{c} \mid \text { if } p^{b} y \in T_{c} \text { then } b \leqq \beta, y p \text {-primitive }\right\}
$$

Then

Let

$$
\left|T_{c}\right| \geqq \sum_{p^{\beta} \sum_{y \in V_{c}}} p^{\beta} \text { and } B\left(\Lambda\left(\mu^{\prime}\right), T_{c}\right)=B\left(\Lambda\left(\mu^{\prime}\right), V_{c}\right) \text {. }
$$

$$
\begin{aligned}
a=p^{(\gamma-1)(n-2)}= & \mid B\left(\Lambda\left(\mu^{\prime}\right), x \mid \text { for any } p \text {-primitive } x * \Lambda\left(\mu^{\prime}\right) .\right. \\
\left|T_{c}\right| \geqq \sum_{p \beta_{y} \in V_{c}} p^{\beta} & =\sum_{p_{y} \in V_{c}} \frac{\left|B\left(\Lambda\left(\mu^{\prime}\right), p^{\beta} y\right)\right|}{a}=\frac{1}{a}\left|B\left(\Lambda\left(\mu^{\prime}\right), V_{c}\right)\right| \\
& =\frac{1}{a}\left|B\left(\Lambda\left(\mu^{\prime}\right), T_{c}\right)\right|=\frac{1}{a}\left|\Lambda\left(\mu^{\prime}\right)\right|=p^{\gamma-1} .
\end{aligned}
$$

(c) By (a) and (b), $\left|T_{*}\right| \geqq \ell p^{\gamma-1}$. Since $\left|M_{c}\right|=p^{n-2}$,

$$
|Q|=\ell p^{n-2} \text {. }
$$

Because $P$ is the disjoint union of $Q$ and $R$, and

$$
|P|=p^{n-1}
$$

we have

$$
|R|=p^{n-1}-\ell p^{n-2} .
$$

This completes the proof of Lemma 1 .

$$
\text { Of course } T \backslash T_{*} \text { denotes }\left\{x \in T \mid x \notin T_{*}\right\} \text {. }
$$

Lemma 2. (a) $|A(P, x)|=p^{n-2}$ for any $x \in T$. If $x \in T \backslash T_{*}$, $y \in T_{*}$ then

(b) $|A(P, x) \cap A(P, y)|=p^{n-3}$ and

(c) the number of $\Lambda\left(\mu^{\prime}\right) \in R$ with $x * \Lambda\left(\mu^{\prime}\right)$ is

$$
|A(R, x)|=p^{n-2}-\ell p^{n-3}, \iota=\left|Q^{\prime}\right| \text {. }
$$

Proof. (a) If $x \in T$ and $x * \Lambda_{r}$ then $\left(x_{2}, \cdots, x_{n}, p\right)=1$ implies there are $p^{n-2}$ choices for $\mu_{2}^{\prime}, \cdots, \mu_{n}^{\prime}$.

(b) follows from the fact that $x \neq \equiv y(p)$ and $\Lambda_{r}$ is fixed.

(c) If $y \in T_{*}$ then $y \in T_{c}$ for a unique $c$. By Lemma 1(a) $A(P, y)=$ $A\left(M_{c}, y\right) \subset M_{c}$ and counting shows $A(P, y)=M_{c}$. Now it is easy to 
see that $|A(Q, x)|=\ell p^{n-3}$. Since $P$ is the disjoint union of $Q$ and $R,|A(R, x)|=|A(P, x)|-|A(Q, x)|=p^{n-2}-\ell p^{n-3}$. This completes the proof of Lemma 2 .

In Theorem 3 if $\gamma=2, \lambda^{\circ}=[1,0, \ldots, 0]$, then $x \in T$ must satisfy the congruence

$$
x_{1}+p \sum_{2}^{n} \lambda_{i} x_{i} \equiv 0\left(p^{2}\right)
$$

for some $\lambda_{2}, \cdots, \lambda_{n}$. Thus

$$
x_{1} \equiv 0(p), x_{1}=p w_{1} \quad \text { for some } w_{1},
$$

and

$$
w_{1}+\sum_{2}^{n} \lambda_{i} x_{i} \equiv 0(p)
$$

so $\left(x_{2}, \cdots, x_{n}, p\right)=1$ and $x * \Lambda_{1}$.

Lemma 3. Suppose $n \geqq 3$ and for each $\lambda=\left[1, \lambda_{2}, \cdots, \lambda_{n}\right]$ the congruence

$$
w_{1}+\sum_{2}^{n} \lambda_{i} x_{i} \equiv 0(p)
$$

has a solution $x \in T$, where $T$ is a stellar set of points, such that if $x \in T$ and for some integer $m$

$$
x=m\left(w_{1}, x_{2}, \cdots, x_{n}\right) \text { then g.c.d. }\left(x_{2}, \cdots, x_{n}, p\right)=1 .
$$

Denote $\widetilde{x}=\left(x_{2}, \cdots, x_{n}\right)$.

Let

$$
T\left(y_{0}\right)=\left\{m y \in T \mid \widetilde{y} \equiv \widetilde{y}_{0}(p), m=1,2,3, \cdots\right\} \text { for some p-primitive }
$$

$y_{0}$.

Then either ( i ) $|T| \geqq\left|T\left(y_{0}\right)\right| \geqq p$ for some $y_{0} \in T$

$$
\text { or (ii) }|T| \geqq p+\max (|T(y)|, 1) \text { for all } y \in T \text {. }
$$

Proof. If $\left|T\left(y_{0}\right)\right| \geqq p$ for some $y_{0} \in T$ we are done. Assume $|\mathrm{T}(y)|<p$ for all $y \in T$. Then $T$ is a $p$-primitive set since $p^{\beta} y \in T$ implies

$$
y, 2 y, \cdots, p^{\beta} y \in T(y) .
$$

$T \neq \varnothing$ implies $T\left(y_{0}\right) \neq 0$ for some $y_{0} \in T$.

Some calculations show, if $y \in T \backslash T\left(y_{0}\right)$, then

(a) $\left|\Lambda_{1} \backslash B\left(\Lambda_{1}, T\left(y_{0}\right)\right)\right|=p^{n-1}-\left|T\left(y_{0}\right)\right| p^{n-2}$,

(b) $\left|B\left(\Lambda_{1}, y\right) \backslash\left\{B\left(\Lambda_{1}, y\right) \cap B\left(\Lambda_{1}, T\left(y_{0}\right)\right)\right\}\right|=p^{n-2}-\left|T\left(y_{0}\right)\right| p^{n-3}$. 
If $y^{j}=\left(y_{1}^{j}, \cdots, y_{n}^{j}\right), j=1,2$ are two distinct points in $T \backslash T\left(y_{0}\right)$ then

$$
\left|B\left(\Lambda_{1}, y^{1}\right) \cap B\left(\Lambda_{1}, y^{2}\right)\right|=\left\{\begin{array}{l}
0 \text { if } y_{i}^{1}=y_{i}^{2} \text { for all } i>1 \\
p^{n-3} \text { otherwise }
\end{array}\right.
$$

Substituting the above, together with (a) and (b), in

$$
\sum_{y \in T \backslash T\left(y_{0}\right)}\left|B\left(\Lambda_{1}, y\right) \backslash\left\{B\left(\Lambda_{1}, y\right) \cap B\left(\Lambda_{1}, T\left(y_{0}\right)\right)\right\}\right|=\sum_{\lambda \notin B\left(\Lambda_{1}, T\left(y_{0}\right)\right)} 1
$$

gives

$$
|T|-\left|T\left(y_{0}\right)\right| \geqq p
$$

3. Proof of Theorem 3. We prove Theorem 3 by induction on $\gamma$. The case $\gamma=2$ was settled in Lemma 3 where we noted satisfying the congruences $\left(\bmod p^{2}\right)$ was equivalent to covering $\Lambda_{1}$. Similarly satisfying the congruences $\left(\bmod p^{\gamma+1}\right)$ is equivalent to covering $\Lambda_{r}$. The $\lambda \in \Lambda_{1}$ play a similar role to the $\Lambda_{r-1}\left(\mu^{\prime}\right) \subset \Lambda_{\gamma}$; (a) and (b) in Lemma 3 play a similar role to (5) and (6) in Theorem 3.

We assume Theorem 3 true for some $\gamma \geqq 2$ and will show it holds for $\gamma+1$. Thus we will be concerned with covering $\Lambda_{r}$, and shall consider it in terms of the $\Lambda_{r-1}\left(\mu^{\prime}\right) \subset \Lambda_{r}$. We must distinguish two cases:

Case 1. $p^{r}>\left|T_{*}\right| \geqq p^{r-1}$.

Recall the families $Q^{\prime}, R^{\prime}, Q, R$ and $P$ defined in $\S 2$. $\Lambda\left(\mu^{\prime}\right) \in R$ implies

$$
B\left(\Lambda\left(\mu^{\prime}\right), T_{*}\right) \neq \Lambda\left(\mu^{\prime}\right)
$$

and the induction implies the number of points of $T$ in $\Lambda\left(\mu^{\prime}\right)$ is

$$
|T| \geqq p^{\gamma-1}+\max \left(\left|T_{*}\right|, p^{\gamma-2}\right) \text { for each } \Lambda\left(\mu^{\prime}\right) \in T \text {. }
$$

In other words, at least $p^{\gamma-1}$ points of $T \backslash T_{*}$ are in each $\Lambda\left(\mu^{\prime}\right) \in R$. Combining

$$
\sum_{x \in T \backslash T *}|A(R, x)|=\sum_{\Lambda\left(\mu^{\prime}\right) \in R}\left|\left\{x \in T \backslash T_{*} \mid x * \Lambda\left(\mu^{\prime}\right)\right\}\right|
$$

with (5) and (6) gives

$$
|T|-\left|T_{*}\right| \geqq p^{r}
$$

Case 2. For all $x \in T, p^{\gamma-1}>|T(x)|$.

By induction, the cardinality of the subset of points of $T$ that covers $\Lambda\left(\mu^{\prime}\right) \in P$ is greater than or equal to $p^{\gamma-1}+p^{\gamma-2}$.

Notice that $|P|=p^{n-1}$.

Lemma 2 (a) gives $|A(P, x)|=p^{n-2}$.

We have 


$$
\sum_{x \in T}|A(P, x)|=\sum_{\Lambda\left(\mu^{\prime}\right) \in P}\left|\left\{x \in T \mid x * \Lambda\left(\mu^{\prime}\right)\right\}\right|
$$

so that

$$
|T| \geqq p^{\gamma}+p^{\gamma-1}
$$

4. Proof of Theorems 1 and 2. As remarked earlier, it is sufficient to prove Theorem 2 in order to conclude Theorem 1. Thus we shall prove only Theorem 2. By [2] and [3] we may assume $n \geqq 3$ and $\alpha \geqq 2$.

We apply Theorem 3 with $\alpha=\gamma \geqq 2$. Thus we have a result about covering the $\Lambda_{\alpha-1}(\mu) \subset V_{\alpha}{ }^{*}$.

Let $N=\left\{\Lambda_{\alpha-1}(\mu) \mid \Lambda_{\alpha-1}(\mu) \subset V_{\alpha}^{*}\right\}$. The number of $\Lambda_{\alpha-1}(\mu) \subset V_{\alpha}^{*}$ is $|N|=1+p+\cdots+p^{n-1}$ and $|A(N, x)|=1+p+\cdots+p^{n-2}$ for any $x \in S$.

We consider two cases corresponding to those in Theorem 3.

Case 1. $\left|T_{*}\right| \geqq p^{\alpha-1}$. Then

Let $M=\left\{\Lambda_{\alpha-1}(\mu) \in N \mid B\left(\Lambda_{\alpha-1}(\mu), T_{*}\right)=\varnothing\right\}$.

$$
|M|=|N|-\left|A\left(N, T_{*}\right)\right|=p^{n-1} .
$$

By Theorem 3 each $\Lambda_{\alpha-1}(\mu) \in M$ will need at least $p^{\alpha-1}$ points of $S \backslash T_{*}$ to be covered by $S$.

If $x \in S \backslash T_{*}, y \in T_{*}$ then

$$
|A(N, x) \cap A(N, y)|=1+p+\cdots+p^{n-3} .
$$

Thus

$$
|A(M, x)|=|A(N, x)|-|A(N, x) \cap A(N, y)|=p^{n-2} \text {. }
$$

Now

$$
\sum_{x \in S \backslash T *}|A(M, x)|=\sum_{\Lambda_{\alpha-1}(\mu) \in M}\left|\left\{x \in S \backslash T_{*} \mid x * \Lambda_{\alpha-1}(\mu)\right\}\right|
$$

so by Theorem 3

$$
\left(|S|-\left|T_{*}\right|\right) p^{n-2} \geqq p^{n-1} p^{\alpha-1}
$$

and the result follows.

Case 2. For all $x \in S, p^{\alpha-1}>|T(x)|$.

By Theorem 3, to cover each $\Lambda_{\alpha-1}(\mu) \in N$ will require at least $p^{\alpha-1}+p^{\alpha-2}$ points of $S$. We have

$$
\sum_{x \in S}|A(N, x)|=\sum_{\Lambda_{\alpha-1}(\mu) \in N}\left|\left\{x \in S \mid x * \Lambda_{\alpha-1}(\mu)\right\}\right| .
$$


$|S|\left(1+p+\cdots+p^{n-2}\right) \geqq\left(1+p+\cdots+p^{n-1}\right)\left(p^{\alpha-1}+p^{\alpha-2}\right)$ and the theorem follows.

5. Bounds. Our bounds in Theorem 2, 3 and Lemma 1 are the best possible in the sense that we can exhibit sets of minimum cardinality which satisfy the conditions. For Theorem 2 let

$$
S=\left\{(x, 1,0, \cdots, 0) \mid 1 \leqq x \leqq p^{\alpha}\right\} \cup\left\{(1, p x, 0 \cdots, 0) \mid 1 \leqq x \leqq p^{\alpha-1}\right\} .
$$

Then

$$
|S|=p^{\alpha}+p^{\alpha-1}
$$

and $S$ satisfies all the congruences. Notice that $S$ is composed of $p+1$ disjoint sets $T(x)$, each of cardinality $p^{\alpha-1}$. We expect this because of the strict inequality in Case 2 of the proof of Theorem 2, as compared with the inequality in Case 1.

For Theorem 3 we exhibit a $T\left(x^{\circ}\right)$ of cardinality $p^{\gamma-1}$ and a $T$ of cardinality $p^{\gamma-1}+p^{\gamma-2}$ containing no $T(x)$ of cardinality greater than $p^{\gamma-2}$. Without loss of generality, let $\lambda^{\circ}=[1,0, \ldots, 0]$.

$$
\begin{aligned}
T\left(x^{\circ}\right)= & \left\{(x p, 0, \cdots, 0,1) \mid 1 \leqq x \leqq p^{\gamma-1}\right\} \\
T= & \left\{(0, \cdots, 0, x p+c, 1) \mid 1 \leqq x \leqq p^{\gamma-2}, 1 \leqq c \leqq p\right\} \cup \\
& \left\{(0, \cdots, 0,1, x p) \mid 1 \leqq x \leqq p^{\gamma-2}\right\}
\end{aligned}
$$

All the congruences of Theorem 3 are clearly satisfied by each of these sets.

Finally for Lemma 1 let $c$ be fixed and

$$
T_{c}=\left\{\left(p^{\alpha-r_{c}}+p^{\alpha-y+1} x, 0, \cdots, 0,1\right) \mid 1 \leqq x \leqq p^{\gamma-1}\right\} .
$$

Then

$$
\left|T_{c}\right|=p^{\gamma-1}
$$

and

$$
B\left(\Lambda\left(\mu^{\prime}\right), T_{c}\right)=\Lambda\left(\mu^{\prime}\right) \text { for all } \Lambda\left(\mu^{\prime}\right) \in M_{c} .
$$

The author wishes to thank Dr. G. K. White for his advice and encouragement in the preparation of this paper.

\section{BIBLIOGRAPHY}

1. J. W. S. Cassels, On the subgroups of infinite abelian groups, J. London, Math. Soc. 33 (1958), 281-4.

2. C. A. Rogers, The number of lattice points in a star body, J. London, Math. Soc. 26 (1951) 307-310. 
L. F. HARRIS

3. G. K. White, On subgroups of fixed index, Pacific J. Math. 28 (1969), 225-232. Received June 16, 1969.

UNIVERSITY OF BRITISH COLUMBIA

VANCOUVER 


\section{PACIFIC JOURNAL OF MATHEMATICS}

\section{EDITORS}

H. SAmelson

Stanford University

Stanford, California 94305

RICHARd PieRCe

University of Washington

Seattle, Washington 98105

\author{
J. DugundJI \\ Department of Mathematics \\ University of Southern California \\ Los Angeles, California 90007 \\ RICHARD ARENS \\ University of California \\ Los Angeles, California 90024
}

ASSOCIATE EDITORS
E. F. BECKENBACH
B. H. NeumanN
F. WOLF
K. YosHIDA

\section{SUPPORTING INSTITUTIONS}

UNIVERSITY OF BRITISH COLUMBIA

CALIFORNIA INSTITUTE OF TECHNOLOGY

UNIVERSITY OF CALIFORNIA

MONTANA STATE UNIVERSITY

UNIVERSITY OF NEVADA

NEW MEXICO STATE UNIVERSITY

OREGON STATE UNIVERSITY

UNIVERSITY OF OREGON

OSAKA UNIVERSITY

UNIVERSITY OF SOUTHERN CALIFORNIA
STANFORD UNIVERSITY

UNIVERSITY OF TOKYO

UNIVERSITY OF UTAH

WASHINGTON STATE UNIVERSITY

UNIVERSITY OF WASHINGTON

AMERICAN MATHEMATICAL SOCIETY CHEVRON RESEARCH CORPORATION TRW SYSTEMS

NAVAL WEAPONS CENTER 


\section{Pacific Journal of Mathematics}

\section{Vol. 35, No. $1 \quad$ September, 1970}

B. D. Arendt and C. J. Stuth, On the structure of commutative periodic semigroups ..................................... 1

B. D. Arendt and C. J. Stuth, On partial homomorphisms of semigroups .... 7

Leonard Asimow, Extensions of continuous affine functions ............ 11

Claude Elias Billigheimer, Regular boundary problems for a five-term recurrence relation ................................... 23

Edwin Ogilvie Buchman and F. A. Valentine, A characterization of the

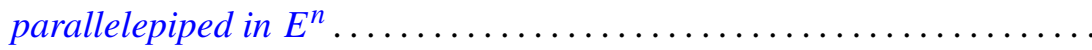
53

Victor P. Camillo, A note on commutative injective rings ............ 59

Larry Jean Cummings, Decomposable symmetric tensors ............. 65

J. E. H. Elliott, On matrices with a restricted number of diagonal values ... 79

Garth Ian Gaudry, Bad behavior and inclusion results for multipliers of type

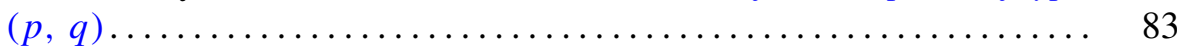

Frances F. Gulick, Derivations and actions .................. 95

Langdon Frank Harris, On subgroups of prime power index . . . . . . . . 117

Jutta Hausen, The hypo residuum of the automorphism group of an abelian

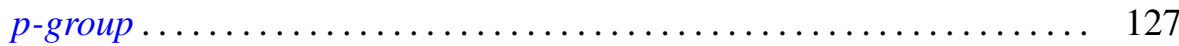

R. Hrycay, Noncontinuous multifuctions . ................... 141

A. Jeanne LaDuke, On a certain generalization of $p$ spaces ............ 155

Marion-Josephine Lim, Rank preservers of skew-symmetric matrices...... 169

John Hathway Lindsey, II, On a six dimensional projective representation of the Hall-Janko group ................................... 175

Roger McCann, Transversally perturbed planar dynamical systems . . . . . 187

Theodore Windle Palmer, Real $C^{*}$-algebras ................... 195

Don David Porter, Symplectic bordism, Stiefel-Whitney numbers, and a

Novikov resolution ....................................... 205

Tilak Raj Prabhakar, On a set of polynomials suggested by Laguerre

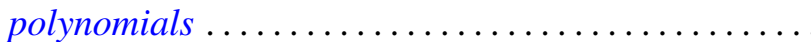

B. L. S. Prakasa Rao, Infinitely divisible characteristic functionals on locally convex topological vector spaces...................

John Robert Reay, Caratheodory theorems in convex product structures ...

Allan M. Sinclair, Eigenvalues in the boundary of the numerical range. .

David R. Stone, Torsion-free and divisible modules over matrix rings ....

William Jennings Wickless, A characterization of the nil radical of a

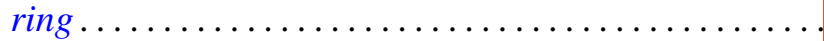

